\title{
Review: evidence on the effectiveness of interventions to assist patient adherence to prescribed medications is limited
}

\author{
McDonald HP, Garg AX, Haynes RB. Interventions to enhance patient adherence to medication prescriptions: scientific \\ review.JAMA 2002;288:2868-79. \\ QUESTION: In non-addicted patients with medical or mental disorders, are \\ interventions designed to assist adherence to self administered prescribed medications \\ effective?
}

\section{Data sources}

Studies were identified by searching Medline, CINAHL, PsycLIT, SOCIOFILE, IPA, EMBASE/Excerpta Medica, and the Cochrane Library (all from 1967 to August 2001). Bibliographies of relevant articles were reviewed, and authors of included studies were contacted for additional trials.

\section{Study selection}

Studies were selected if they were unconfounded randomised controlled trials (RCTs) of interventions to improve adherence with self administered prescribed medications for a medical or psychiatric disorder, measured both medication adherence and treatment outcome, had $\geq 80 \%$ follow up of each group studied, and the duration of follow up for studies with positive initial findings was $\geq 6$ months.

\section{Data extraction}

Data were extracted on sample size, details of intervention strategies for adherence, details of treatment for the underlying medical or mental disorder, study quality, and outcomes (adherence rates and patient outcomes).

\section{Main results}

33 RCTs met the selection criteria. These trials evaluated 39 unconfounded interventions. Adherence interventions were tested alone and in combination, with common themes such as more instruction for patients (oral and written material and programmed learning); increased communication and counselling (eg, compliance therapy and family intervention); increased convenience of care (eg, provision at the worksite and simplified dosing); more involvement of patients in their care through self monitoring of disease status; reminders (eg, reminder pill packaging); and reinforcement or rewards for both improved adherence and treatment response (eg, reduced frequency of visits and partial payment for blood pressure monitoring equipment). Conditions studied included hypertension (8 RCTs); schizophrenia or acute psychosis (8 RCTs); asthma, chronic obstructive pulmonary disease, or both (5 RCTs); depression (2 RCTs); human immunodeficiency virus (2 RCTs); diabetes (2 RCTs); rheumatoid arthritis (1 RCT); epilepsy (1 RCT); and hyperlipidaemia and cardiovascular disease (1 RCT).

$49 \%$ of interventions tested were associated with statistically significant increases in medication adherence, and $44 \%$ reported statistically significant improvements in treatment outcome. Adherence interventions that were effective were complex, including combinations of more convenient care, information, counselling, reminders, self monitoring, reinforcement, family therapy, and other forms of additional supervision or attention. Simple interventions that showed some success included simplified dosing regimens for those taking antihypertensive and lipid lowering medications and counselling about the importance of full adherence to antibiotic regimens reinforced by written instructions.

\section{Conclusion}

In patients with medical or mental disorders, limited evidence suggests that several interventions designed to assist patients' adherence to prescribed medications may be effective.
Sources of funding:

Population Health

Information Project;

Canadian Institutes of

Health Research;

Kidney Foundation of

Canada.

For correspondence:

Dr R B Haynes,

McMaster University

Medical Centre,

Hamilton, Ontario,

Canada.

bhaynes@mcmaster.ca

Abstract and

commentary also

appear in ACP Journal Club.

\section{COMMENTARY}

Healthcare providers tend to see their job as making decisions about care and the patient's job as carrying them out. Yet, those of us who have been prescribed daily medications have found it very difficult to comply with fitting them into our daily routines, especially for asymptomatic conditions. This difficulty is exacerbated by the increasing complexity of treatment regimens resulting from controlled trials (where there is usually great effort to maximize drug adherence), which continue to show us additional drugs that improve outcomes and those drugs are subsequently recommended for routine use. For example, 20 years ago drug therapy for heart failure consisted of digitalis and diuretics. Now, managing this condition takes $4-5$ drugs, guided by a complex algorithm. ${ }^{1}$ No wonder compliance is so difficult for both physicians and their patients.

The good news from the systematic review by McDonald $e t$ al is that several interventions, many of which are relatively easy to invoke, can increase patient adherence to drug regimens. The bad news is that it often takes efforts by several people to effect this adherence. And worse, neither private nor public insurance pays for these extra efforts. However, many of the interventions aimed at improving quality of care and patient safety ${ }^{2}$ can also be used to enhance medication adherence. If a commitment exists to enhance drug adherence as part of good prescribing, then everyone involved in providing care (eg, physicians, nurses, pharmacists, mental health counsellors, and administrators and technologists who design and maintain health care systems) should take a good, long look at the review by McDonald $e t$ al to see where they can contribute. William M Tierney, MD Indiana University School of Medicine and the Regenstrief Institute, Inc. Indianapolis, Indiana, USA

1 Hunt SA, Baker DW, Chin MH, et al. ACC/AHA Guidelines for the Evaluation and Management of Chronic Heart Failure in the Adult: Executive Summary A Report of the American College of Cardology/Ame 1995 Guidelines for the Fre on Practice Guidelines (Cof Managen (Cof America. Circulation 2001;104:2996-3007.

Institute of Medicine (US). Committee on quality of Health Care in America. Crossing the quality chasm: a new health system for the 21 st century/Committee on Quality Health care in http://www.nap.edu/books/0309072808/html/ 